\title{
Combatting Childhood Obesity in An Australian Community Intervention: Do Goal-Setting and Incentives Encourage Healthy Eating and Exercise Behaviour Change?
}

Gemma Enright ${ }^{1,2}$, Alex Gyani ${ }^{3,7}$, Margaret Allman-Farinelli ${ }^{4}$, Chris Rissel $^{5}$, Christine Innes-Hughes ${ }^{5}$, Sarah Lukeis $^{6}$, Anthony Rodgers ${ }^{2}$, Lily Chen ${ }^{2}$ and Julie Redfern ${ }^{1,2 *}$

${ }^{1}$ Faculty of Medicine and Health, University of Sydney, Australia

${ }^{2}$ The George Institute for Global Health (Cardiovascular Division), Australia

${ }^{3}$ NSW Department of Premier and Cabinet, Behavioural Insights Team, Australia

${ }^{4}$ Charles Perkins Centre, University of Sydney, Australia

${ }^{5}$ Ministry of Health, NSW Office of Preventive Health, Australia

${ }^{6}$ The Better Health Company, Australia

${ }^{7}$ Behavioural Insights Team, Australia

Submission: March 23, 2019; Published: April 23, 2019

*Corresponding author: Julie Redfern, Faculty of Medicine and Health, University of Sydney, The George Institute for Global Health (Cardiovascular Division), The University of Sydney at Westmead Hospital, PO Box 154 Westmead NSW 2154, Australia

\begin{abstract}
This research aimed to explore the contextual influences and behaviour change techniques on diet and exercise behaviours in overweight or obese children aged 7 to 13yrs. Qualitative methodology comprised 18 family interviews with families participating in an Australian community weight-management program during a cluster randomised controlled trial investigating goal-setting and incentives for enhancing healthy eating and exercise behaviours. Inductive thematic analysis and the Behaviour Change Technique Taxonomy guided analyses.

This research demonstrated that parents can have different approaches and levels of confidence in managing healthy behaviour in their families but share similar emotional struggles that inhibit healthy eating and exercise in the family. This evaluation, combined with existing psychological literature, supports the concept of creating autonomous accountability around goal-setting as the focus of future weight management programs, which is potentially imperative to long-term behaviour change. Future interventions targeting health-related behaviour change should aim to avoid controlling approaches that detract from participants' sense of control, self-efficacy and support.
\end{abstract}

Keywords: Childhood-obesity; Public-health; Incentives; Behavior-change; Qualitative; Prevention

Abbrevations: BCT: Behavior Change Technique; cRCT: Cluster Randomised Controlled Trial

\section{Introduction}

Reducing childhood obesity is of increasing public health importance. In 2014, 41 million children under five years of age were estimated to be overweight [1] and being overweight as a child has been shown to carry a high risk of being obese as an adult [2] and accelerating the risks of associated conditions such as cardiovascular disease [3]. As such, public health services are integral in preventing and managing childhood obesity

[4], and strategies informing interventions for health-related behaviour change in children are increasingly relevant at policy level. Systematic reviews investigating the effectiveness of childfocused behavioural interventions have indicated small positive outcomes on healthy eating and physical activity behaviours [5], however, facilitating behaviour change in obesity-focused public health initiatives is often challenging because people struggle 
to establish healthy habits they can sustain after the program [6]. This is despite evidence for specific behaviours required for effective weight-loss [7-12] and elements recognised as important to achieve long-term behaviour changes $[7,12]$.

Evidence is mounting in adults for a role of incentives in enhancing health-related behaviour change in the short-term [13-20], but high heterogeneity across study designs, incentive strategies and outcome measures, and a lack of long-term follow up have prevented firm conclusions on the most effective incentive strategy for behaviour change. The studies also highlighted that existing incentive-based interventions in natural settings tend to lack grounding in basic behavioural theories. Preliminary evidence for incentives enhancing health-related behaviour change in children is encouraging, though sparse and low quality. A recent review [21] highlighted several (uncontrolled) studies that found positive results associated with incentivising health behaviours in children, with two studies reporting sustained effects at two months [22] and six months [23] follow-up. Several small randomised controlled trials (RCTs) have used a combination of psychological strategies (such as peer modelling) and low value incentives to encourage behaviour change in children within primary schools to encourage exercise behaviour $[24,25]$ and fruit and vegetable consumption [26,27]. These studies highlighted that incentive strategies shown to effectively influence multiple target behaviours short term may have varied effects longer term for those behaviours, and that differences can emerge by age, gender and socio-economic background [22,26]. Information on the social and environmental influences and behavioural mechanisms involved in health-related behaviour change is missing from existing research on child-focused incentive-based interventions in community settings.

RCTs are important for establishing the effectiveness of community-based interventions but are unable to provide policy-relevant information on how complex interventions work in context [28]. Conducting qualitative research alongside an RCT provides rich contextual information about what worked and did not work, the type of change that occurred, and the circumstances in which it was most and least effective and why [29-31]. Such information can inform strategies for specific child populations and facilitate adoption of intervention components into community health settings. This study aimed to determine the contextual factors and active behaviour change components influencing the behavioural outcomes of an incentive-based behaviour change strategy targeting child obesity.

Specifically, the research aimed to understand

i. Attitudinal characteristics of participating families;

ii. Factors influencing impacts of the strategy over 18-months

iii. Active behaviour change techniques in the strategy and links with psychological theory.

\section{Material and Methods}

\section{Design}

Qualitative research conducted in New South Wales Australia between 2014-2016 between the six and 18-month follow-up of an associated cluster randomised controlled trial (cRCT) [32]. In brief, the cRCT ( $\mathrm{n}=512$ children, 38 program sites, five local health districts) evaluated a goal-setting linked to incentives strategy for enhancing and sustaining healthy eating and exercise behaviour change in overweight and obese children. The cRCT was set within the context of 'Go4Fun'; an existing Australian evidence-based community weight-management program [33]. The study was approved by the South West Sydney Human Ethics Committee (HREC/14/LPOOL/480), and site-specific approvals were obtained from research governance offices in participating local health districts.

\section{Participants and Recruitment}

A total of 18 parents ( $n=24$ children) took part in 14 family interviews and 1 group parent-only interview. The mean age of participants' children was $10( \pm 2)$ years, $16 / 24$ were female and the mean BMIz score of the children was $2.0 \mathrm{~kg} / \mathrm{m}^{2}( \pm 0.46)$. $12 / 24$ children spoke English as their primary language at home, and $4 / 24$ were from solo parent households. Participants were selected from families already participating in the cRCT (treatment and control) who consented to further research participation. Children were aged 7-13yrs at the time of recruitment, had a body mass index greater than the 85th percentile for their age and gender [34] and met the criteria to participate in the community weight-management program at participating sites [32]. Informed written consent was obtained from all study participants' parents/ carers, and all participants were unaware which components of the weight management program were part of the trial vs the standard program. Families were recruited for qualitative research via a survey of all parents administered at the six-month follow-up assessment of the cRCT. Participating families consented to participate in either a focus group or family interview, and maximum variation sampling was used to select participants based on family and program characteristics; high ( $\geq 60 \%$ of sessions) and low attendance ( $\geq 0 \%$ of sessions), single child and multiple sibling families, small ( $<$ nine children) and large (>ten children) programs. Varied ethnicity, household, cultural and linguistic diversity (CALD) and socioeconomic status characteristics were also included.

\section{Data Sources}

The dataset comprised family interviews conducted with individual parents, groups of parents or parents accompanied by their children who participated in the weight management program. All interviews took place between March and June 2017, followed a semi-structured approach aided by discussion guides to facilitate natural conversation, and lasted approximately 1.5 hours. Interviews were audio recorded and confidentiality was agreed with participants prior to the interview. 


\section{Analysis and Interpretation}

An inductive thematic analysis anchored in a constructionist theoretical framework was carried out, using a 6-step guideline framework for thematic analysis [35]. This approach allowed for theoretical flexibility, given the aim was to explore participants' described experiences of the community program, as well as the wider context of their lives on potential influences on healthy behaviour. The analysis therefore aimed to produce latent themes. Family interviews were transcribed verbatim by GE to familiarise and then sort the data into initial concepts. Each transcript was scanned, systematically coded and organised into themes by GE. A second researcher then independently coded the transcripts, undertaking a reflexive dialogue to check whether extracts confirmed or contradicted the identified themes. A constant comparison method was used, where codes and themes were collapsed and expanded until no new themes were found. Themes were defined and reviewed amongst the wider research group, and disagreements were resolved by discussion.

Previous work details how intervention components were developed for the cRCT [36]. In brief, the research team identified behaviour change concepts which informed the design of a goalsetting process linked to an incentives and rewards scheme for goal completion. This scheme was supported by additional theorybased intervention components including; a visual handout to help families plan incremental goals towards a bigger health outcome, a goals contract to encourage commitment to goals, a group tracking chart to publicly track progress, and a post-program lottery prize incentive with text message prompts for six-months after the weight-management program. The Behaviour Change Technique Taxonomy (BCTTv1) [37] provided a framework for describing what was delivered by each of the cRCT components, and helped to draw out active, non or less-active behaviour change techniques (BCTs) within the cRCT components [38].

Transcripts were scanned and coded specifically for extracts relating to the influence of each cRCT component. 'Goal achievement' and motivation towards goal achievement, as reported by participants, was used as a proxy for healthy eating and exercise behaviour on which to evaluate the 'activeness' of the identified BCTs. Inferential links were made between parents' accounts of their engagement with the intervention components and the associated BCTs identified in those intervention components. A separate document was used with the cRCT components as headings to guide the process, Using the taxonomy enabled this evaluation to build on an increasingly accepted common language for recognising and specifying components of behavioural interventions that are effective in influencing obesityrelated behaviours (use of the taxonomy supports the CONSORT guidelines for the reporting of behaviour change interventions [39]. BCTTv1 online training was undertaken to maximise practical proficiency in recognising and coding BCTs according to the definitions used in the taxonomy.

\section{Results and Discussion}

\section{Attitudinal Characteristics of Participating Families (Aim 1)}

Attitudes towards Health in the Family

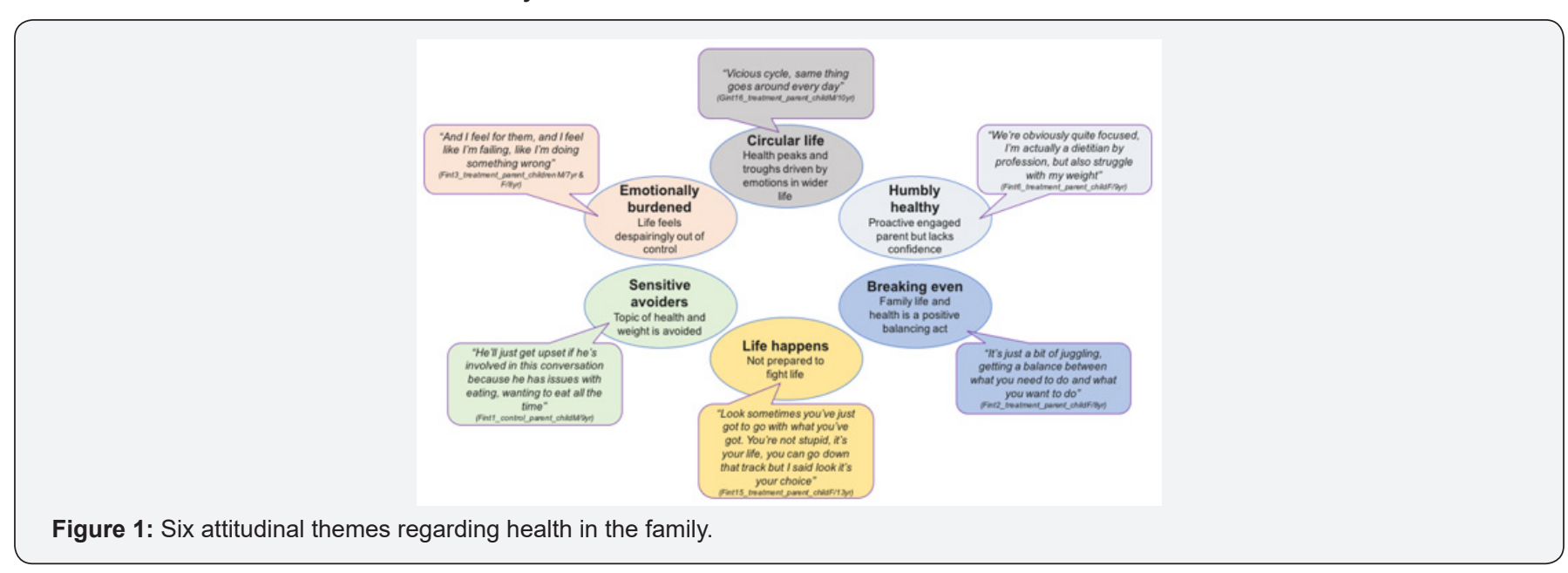

Table 1: Attitudinal themes.

Self-Blame

"I'm to blame. Sometimes I sit here and try and break it down in my head and I think am I really doing something this wrong?" (Fint3_treatment_ parent_children M/7yr \& F/8yr emotionally burdened)

"I blame the stress with me and that's when the kids get the bad habits. I'd eat anything and do what I like, and you see the kids copying your behaviour" (Gint18_treatment_parent_childF/10yr circular life) 


\section{Psychology and Behavioral Science International Journal}

"Yeah, I might cave in, I feel like I always have to be the bad one and everyone else gets to be the good guy"(Fint3_treatment_parent_children M/7yr
\& F/8yr emotionally burdened)
"The problem is its still more in our control, it feels like it's more us constantly on their backs" (Gint15_treatment_parent_childF/13yr life happens)
Fire-Fighting
"If I have a battle it's going to be with homework" (Fint5_treatment_parent_childF/8yr humbly healthy)
"It's hard to be consistent every single day because you've got other stuff going on, you might have a couple of good days and then you'll have a busy
afternoon and they'll take advantage of that" (Gint18_treatment_parent_childF/10yr circular life)
External Sabotage
"Their weight is a lot to do with their diet from their mum. She's still feeding them crap. If you're not on the same page you just struggle more don't
you" (Fint4_treatment_parent_children M/7yr, F/9yr \& M/11yr emotionally burdened)
"It is really hard packing lunches when you know what other kids will be getting. The crap they take to school makes it harder to encourage your
children to be healthy when you know that nobody else is" (Fint11_treatment_parent_childF/9yr circular life)
Overwhelm from Different Family Needs
"She and her twin brother can eat exactly the same thing yet [twin] is a lot smaller" (Fint8_treatment_parent_childF/9yr sensitive avoider)
"I have a son who needs to put weight on, but I've got [participant] so I need to try and balance it out. I give them one thing and it conflicts, it's really
hard" (Gint18_treatment_parent_childF/10yr circular life)

Six attitudinal themes regarding health in the family were found in participating families, which were used to describe the 'types' of families in the study (Figure 1). The emotional experience of the mother regarding caring for her family was central to these attitudes, particularly a lack of confidence and control and a sense of personal failure. Five further attitudinal themes were found (Table 1):

i. 'self-blame'; guilt, failure or regret as a parent,

ii. 'onus on us'; burden and resentment about shouldering responsibility,

iii. 'fire-fighting'; stress due to lack of routine and hectic lifestyle

iv. 'external sabotage'; victimization by external sabotage, and

\section{Attitudes towards Incentivizing and Rewarding children at Home}

Table 2: Attitudes towards incentivising and rewarding children.

Reward Systems are Unsustainable at Home

“It worked fantastic until they both got to their limit of what they wanted to purchase, and they bought their item and it fizzled and they didn't want to wash the dishes anymore". (Fint1_control_parent_children F/8yr \& F/9yr sensitive avoider)

"Not something as a parent, you want to keep doing. Life is full on and I don't have the time to set up a reward system at home" (Fint12_control_ parent_children F/8yrF/9yrF11yr breaking even)

Material Rewards (At Home) does not Align with Values

“I don't think it's necessary. These are things we should be doing anyway" Fint8_treatment_parent_childF/9yr sensitive avoider)

“I don't want my kids to feel entitled, that if I'm gonna do the dishes you need to give me 5 cents. In my culture, it's the opposite; they are not supposed to be rewarded for anything because that'll spoil them" (Fint12_control_parent_children F/8yrF/9yrF11yr breaking even)

Threats are more Effective than Rewards

"It's more about punishments at home, I don't really like rewards, the kids have so much now. It's better to take away privileges rather than rewards" Fint5_treatment_parent_childF/8yr humbly healthy)

"I have to threaten my kids, encouragement does not work".

(Fint10_treatment_parent_childF/11yr emotionally burdened)

Parents have Ingrained Habits around Food Rewards

"When I grew up everything was about a treat, so I'm wired that way and automatically walk past the bakery and see doughnuts and think oh she'd love that"

(Fint1_control_parent_children F/8yr \& F/9yr sensitive avoider) 


\section{Psychology and Behavioral Science International Journal}

"Food was always used as a reward when we were growing up, every week we went for an ice cream. We've got to break our own habits" (Gint18_ treatment_parent_childF/10yr circular life)

Attitudes towards incentivising and rewarding children.

Four themes were found (Table 2)

i. ' 'reward systems are unsustainable at home'; parents felt giving their children money for chores reaches a limit, and on-going reward systems are unrealistic in terms of effort and expense required,

ii. 'material rewards (at home) did not align with values'; for example, in Nigeria praise and encouragement of children was described by one family as more acceptable than giving material rewards,

iii. 'threats are more effective than rewards'; particularly in relation to older children, and

iv. 'parents have ingrained habits around food rewards'; making norms and habits hard to break.

Parents acknowledged a need to 're-wire' their own habits, learn how to give non-food rewards, and re-frame pleasant

Table 3: Long-term impacts of the strategy over 18-months. occasions associated with food. Educating parents about appropriate rewards is an important component to continue incorporating into future weight management programs., however this research did not suggest that reward systems will be adopted at home outside of weight management programs.

\section{Factors Influencing Impacts of the Strategy over 18-months (Aim 2)}

Four themes were identified that reflected the participants' perceived long-term impacts of their participation in the community program;

i. 'retained mindfulness' (of healthy eating and exercise),

ii. 'keenness to continue new routines',

iii. ' healthy eating and exercise as a work in progress' and

iv. 'a decline in healthy eating and exercise behaviour' (Table

$3)$.

\section{Retained Mindfulness}

"We are looking at labels a lot more and they are very active in doing that in the supermarket. I love being able to refer back. That has stayed, that has stayed a lot"

(Fint3_treatment_parent_children M/7yr \& F/8yr emotionally burdened)

“Before go4fun we weren't health conscious at all. It was a good wakeup call. Just enough to create awareness” (Fint12_control_parent_children F/8yrF/9yrF11yr breaking even)

Keenness to Continue Routines Developed During the Program

"I actually realised it was quite fun to have one day when there was a structure around it, so we now do Saturdays and then Sundays are our day we don't have anything pre-organised" (Fint2_treatment_parent_childF/8yr breaking even)

"It's probably why we ended up doing boot camp. I suppose go4fun was what set us looking for another activity that worked well in a group environment that wasn't a team sport" (Fint6_treatment_parent_childF/9yr humbly healthy)

Perception of Healthy Eating and Exercise as A Work in Progress

"You've got to look at the big picture, it's about making sure you're giving them the right tools to eventually take over the responsibility themselves.

So, from that point of view I think that's an on-going work in progress and it certainly gave us the tools" (Fint2_treatment_parent_childF/8yr breaking even)

"It's a life changing experience, not just temporary" (Fint14_treatment_childF/10yr breaking even)

Decline in Healthy Eating and Exercise Behaviour

"It did phase out fairly quickly. Tapered a couple of months afterward. She probably was a little bit more accountable because she was going back each week, she had her goals she worked on each time and that was important to her at the time, where now those specific goals aren't there anymore" (Fint6_treatment_parent_childF/9yr humbly healthy)

"Then she just completely lost interest once the program finished, within 2 months of the program ending she was not eating fruit and veg again". (Fint10_treatment_parent_childF/11yr emotionally burdened)

Parents' perceptions were mixed regarding direct attribution of long-term lifestyle changes to the weight-management program itself. Many parents said their children had 'tapered off' doing the specific goals set during the program and were no longer thinking in terms of 'achieving goals', within two-months of the end of the program (Table 3). However, several parents acknowledged their children had integrated learnings and life skills from the 10 -week program into their daily awareness and described their attitude to health now as a 'work in progress' (Table 3). Some parents were inspired to continue with their weekly commitment to exercise after the program, encouraged by the new routines the goalsetting process had initiated (Table 3). Puberty was also identified by parents as an influential factor on health behaviours in older children, with the transition to high school referred to as a time of disruption and shifting of control over health-related decisionmaking and external influences on their child's health behaviours; 
"We've had some big changes, [participant] is in high school now and his appetite has changed a lot. He has periods where he's more conscious, but since he's started high school it's definitely gone downhill, and peer group pressure" (Gint16_treatment_parent_ childM/10yr circular life)

\section{Behaviour Change Techniques and links with Psychological Theory (Aim 3)}

\section{Active Behaviour Change Techniques}

Table 4 summarises the behavioural theories underpinning the cRCT intervention components, and associated behaviour change techniques (BCTs). The most active BCTs in motivating goal completion during the ten-week program included; 'goal-setting (behaviour)' and 'graded tasks' via focusing on small incremental behavioural goals rather than outcomes, 'action planning' via recording implementation intentions, and 'reviewing behaviour' and 'monitoring of behaviour by others without feedback' via tracking goal progress.

Whilst 'reward and threat' was a core input to the cRCT, it was less motivating than BCTs based on 'goals and planning'. This aligns with a theme found where parents described the rewards as an "added bonus" and more effective in encouraging attendance in the ten weekly sessions than goal completion.

"Setting the goals was an incentive but knowing there was going to be a reward was an added bonus. But that wasn't the focus for her, we looked at the goal itself and that was what she was working on, not so much about the reward" (Fint8_treatment_ parent_childF/8yr)

"If there's any sort of tangible reward at the end that's a bonus for them. It was good but it wasn't the reason why we kept coming back" (Fint6_treatment_parent_childF/9yr)

The interviews indicated that the incentives offered in the cRCT were overall accepted by parents despite their rejection of incentivising and rewarding children at home. This may support the idea that external reward systems (e.g. at school) are more likely to engage children than reward systems at home where the process of learning revolves around a child's intrinsic motivation [41].

Table 4: Behavioural theories, associated intervention components, and identified behaviour change techniques.

\begin{tabular}{|c|c|c|c|}
\hline Behavioural Theory Informing cRCT & Intervention Component Developed for cRCT & $\begin{array}{l}\text { Behaviour Change Technique } \\
\text { Identified }\end{array}$ & $\begin{array}{l}\text { Activity -degree of } \\
\text { Influence on Healthy } \\
\text { Eating and Exercise }\end{array}$ \\
\hline $\begin{array}{l}\text { Incentivising behaviours and activities } \\
\text { rather than outcomes [42-44]. }\end{array}$ & $\begin{array}{l}\text { Task of setting small achievable goals focused } \\
\text { on nutrition and physical activity }\end{array}$ & $\begin{array}{l}\text { Goals and planning: } 1.1 \text { goal } \\
\text { setting (behaviour) }\end{array}$ & ++ \\
\hline \multirow{5}{*}{$\begin{array}{l}\text { An 'implementation intention' can help } \\
\text { people achieve a goal [45-47]. }\end{array}$} & Writing down goals on a goals contract & $\begin{array}{l}\text { Goals and planning: } 1.4 \\
\text { action planning }\end{array}$ & ++ \\
\hline & $\begin{array}{l}\text { Agreeing goals with parents on a goals } \\
\text { contract }\end{array}$ & $\begin{array}{l}\text { Goals and planning: } 1.8 \\
\text { behavioural contract }\end{array}$ & ++ \\
\hline & Setting small achievable goals & $\begin{array}{l}\text { Repetition and substitution: } \\
8.7 \text { graded tasks }\end{array}$ & ++ \\
\hline & $\begin{array}{l}\text { Re-setting and stretching of goals each week } \\
\text { with the program leader via weekly check-ins. }\end{array}$ & $\begin{array}{l}\text { Goals and planning: } 1.5 \\
\text { review behaviour (goals) }\end{array}$ & ++ \\
\hline & $\begin{array}{l}\text { Prompting type of goals set by group leader } \\
\text { via weekly check-ins. }\end{array}$ & $\begin{array}{l}\text { Repetition and substitution: } \\
8.2 \text { behaviour substitution }\end{array}$ & + \\
\hline \multirow{2}{*}{$\begin{array}{l}\text { Individuals are motivated to complete a } \\
\text { goal when they can see their progress [48] } \\
\text { Larger effects on goal achievement can be } \\
\text { expected when outcomes are made public } \\
\text { and when information is recorded [49] }\end{array}$} & $\begin{array}{l}\text { Tracking goal achievement publicly via } \\
\text { recording goal completion with stars on a } \\
\text { group tracking chart }\end{array}$ & $\begin{array}{l}\text { Feedback and monitoring: } \\
2.1 \text { monitoring of behaviour } \\
\text { by others without feedback }\end{array}$ & ++ \\
\hline & $\begin{array}{c}\text { Tracking goals publicly via a group tracking } \\
\text { chart }\end{array}$ & $\begin{array}{l}\text { Comparison of behaviour: } 6.2 \\
\text { social comparison }\end{array}$ & + \\
\hline \multirow{2}{*}{$\begin{array}{l}\text { Small and frequent rewards can increase } \\
\text { task perseverance [48] }\end{array}$} & $\begin{array}{c}\text { Offer of low value weekly rewards for goal } \\
\text { achievement }\end{array}$ & $\begin{array}{l}\text { Reward and threat: } \\
10.1 \text { material incentive } \\
\text { (behaviour) }\end{array}$ & + \\
\hline & $\begin{array}{c}\text { Immediate provision of reward for goal } \\
\text { achievement }\end{array}$ & $\begin{array}{c}\text { Reward and threat: } 10.1 \\
\text { material reward (behaviour) }\end{array}$ & + \\
\hline $\begin{array}{l}\text { Lottery-style rewards may increase } \\
\text { effectiveness of incentives }[13,18]\end{array}$ & $\begin{array}{l}\text { Lottery prize draw incentive for six months } \\
\text { after a } 10 \text {-week weight management program }\end{array}$ & $\begin{array}{c}\text { Reward and threat: } \\
\text { 'anticipation of future reward } \\
\text { (non-certain)' - not listed in } \\
\text { BCTTv1 }\end{array}$ & + \\
\hline $\begin{array}{l}\text { Text message prompts can improve health } \\
\text { behaviours [50] }\end{array}$ & $\begin{array}{l}\text { Weekly SMS goal reminders for six months } \\
\text { after a 10-week weight management program }\end{array}$ & $\begin{array}{l}\text { Reward and threat: } 10.4 \\
\text { social reward }\end{array}$ & + \\
\hline
\end{tabular}

CRCT, cluster randomised controlled trial

++ Strong supporting extracts across dataset relating to the influence of the BCT

+ Weaker supporting extracts across dataset relating to the influence of the BCT 
BCTs that were identified within the cRCT components, but less active in motivating goal-related behaviours included; 'social comparison' and 'social reward' (Table 4). Parents described their children as being more motivated by seeing their own progression than comparing themselves against others, and whilst 'supportive praise' was active via 6-month text message prompts, not all parents engaged with this component. The research team found a BCT that did not fit with BBTTv1 definitions but was inherent in the lottery prize draw cRCT component - 'anticipation of future reward (non-certain)'. Family interviews indicated that anticipation of a larger but uncertain reward (i.e. lottery prize draw) was less active in encouraging behaviour change than anticipation of the frequent weekly incentives and rewards linked directly to goal achievement.

\section{Links with psychological Theory}

A combination of Self-Determination Theory [51] and Social Cognitive Theory [52] could explain the behaviours associated with the cRCT over ten-weeks and 18-months. Self-Determination theory based on Cognitive Evaluation Theory [53] and Organismic Integration Theory [54] describes how extrinsic motivations such as material incentives and rewards can facilitate intrinsic motivation. Intrinsic motivation can be fostered if an activity (e.g. goal-setting) generates a sense of autonomy (choosing to conduct actions that align with own beliefs), competence (self-efficacy or perception of personal achievement) and relatedness (anticipated social interaction or social support) [55]. In the cRCT, parents and children agreed and wrote down the children's goals together via a goals contract helping to satisfy perceived autonomy. Families were provided with weekly tracking and positive feedback on progress via a group tracking chart, and rewards for goal achievement which together helped to satisfy 'competence' (specifically, via creating a sense of progression), and weekly 'check-ins' with program leaders to evaluate goal progress helped to satisfy 'relatedness' (specifically, via perceived social support).

Accountability has emerged in a recent review of health-related psychological literature as a key mediator of the psychological factors necessary for supporting intrinsic (autonomous) motivation and longer-term behaviour change [56]. This theory has strong relevance to this evaluation. Although accountability is classically associated with extrinsic and 'controlled' behaviour, the sense of accountability generated by planning, agreeing and tracking of goals in the ten-week program, was identified by a previous work as key to intervention engagement, and intrinsic motivation [57]. A meta-analysis of three studies (on adults in a professional environment) found 'autonomous motivation' is closely related to goal progress and is mediated by implementation planning [58]. This supports the continued use of implementation intentions as part of goal-setting in future weight-management programs.

Families described their motivation to continue healthy eating and exercise as declining after the ten-week program (Tables 3 \& 4), which coincided with a reduced sense of autonomy, progression and support. Post-program text message prompts were described as 'pushy' (lack of autonomy) and heightened parents' perceived 'onus on us' (lack of support). There was also no visual or personalised feedback on progress after the ten-week program (lack of progress). These combined factors may explain why families no longer felt accountable to their goals and gave up. Many parents reported positive changes in their children at 18-months, including retained awareness of healthy eating and exercise. However, families found translating attitudes into behaviour after the ten-week program challenging, and future interventions may be enhanced by post-program initiatives that support psychological needs related to accountability rather than longer-term extrinsic incentive schemes (such as lottery-prize draws). There is evidence in the literature that 'accountability partners' help people keep a commitment through text [59]. The literature has also identified positive social feedback and praise as highly important in satisfying the need for self-efficacy and enhancing intrinsic motivation [56] which aligns with the parents who reported their children valued praise and recognition for their goal achievement over the material rewards.

\section{Conclusion}

This research demonstrated that parents can have different approaches and levels of confidence in managing healthy behaviour in their families but share similar emotional struggles that inhibit healthy eating and exercise in the family. This evaluation, combined with existing psychological literature, supports the concept of creating autonomous accountability around goal-setting as the focus of future weight management programs, which is potentially imperative to long-term behaviour change. Future interventions targeting health-related behaviour change should aim to avoid controlling approaches that detract from participants' sense of control, self-efficacy and support. Program implementers should also strive to understand and work with participating families to manage inhibiting attitudes and barriers to the balance and control in their lifestyles.

This evaluationincludestheprovision ofqualitativeinformation providing context on influential family characteristics to inform the future design of family-focused health-related behavioural interventions targeting childhood obesity. The information also contributes to understanding the usefulness of behaviour change techniques within an established set of definitions, which will aid replication. Though this research represents views of parents rather than specifically the participating children it highlights the importance of understanding the family as a whole.

Overall, the behaviour change intervention implemented in the associated cRCT was received differently by different families, and factors such as socio-economic status, family size, and cultural norms are likely to influence families' response to future schemes involving goal-setting and incentives. Further exploratory and quantitative work will need to be completed to understand and quantify these factors. 


\section{Acknowledgement}

\section{Author Contributions}

GE led the drafting of all sections of the article in consultation with all the co-authors. GE moderated the interviews and led the analysis and interpretation of themes, with LC also independently coding the transcripts. JR and GE led the application for funding for this work. All authors provided substantial contribution to the concept and design of the evaluation, drafting the protocol paper and reviewing critically for important intellectual content and final approval of the version for publication. GE, AG, MA-F, CR CI$\mathrm{H}, \mathrm{SL}$ and JR also provided substantial contribution to the design and implementation of the associated cRCT.

\section{Additional Acknowledgement}

This evaluation would not have been possible without the contributions of the cRCT investigator team and Working Group. We thank all families and site leaders for their support and work on this study. We also thank Mr Simon Raadsma for his contributions and commitment to the project during his time at the Department of Premier and Cabinet. Investigators and Working Group members who are not co-authors on this paper, and their affiliates are listed below:

i. Office of Preventive Health: Anita Cowlishaw; Santosh Kanal; Nicholas Petrunoff

ii. Behavioural Insights Unit, Department of Premier and Cabinet: Shirley Dang

iii. Western Sydney LHD: Christine Newman; Michelle Nolan; Deborah Benson, Kirsti Cunningham

iv. South Western Sydney LHD: Mandy Williams; Leah Choi; Kate Jesus; Stephanie Baker

v. South Eastern Sydney LHD: Myna Hua; Linda Trotter; Lisa Franco

vi. North Sydney LHD: Paul Klarenaar; Jonothan Noyes; Sakara Branson

vii. Hunter New England LHD: Karen Gillham; Dr John Wiggers; Silvia Ruano-McLerie

viii. Mid North Coast LHD: Ros Tockley; Margo Johnson

ix. Better Health Company; Madeline Freeman; Bec Thorp

x. The George Institute for Global Health; Sarah Eriksson; Caroline $\mathrm{Wu}$

In addition, we thank the Go4Fun program leaders, and representatives from our funding partner organisations, including the Heart Foundation, who have contributed to the development and implementation of the RCT.

\section{Funding}

This research is funded in-kind provided by the George Institute for Global Health, NSW Department for Premier and
Cabinet and NSW Office of Preventive Health. GE is funded by an NHMRC postgraduate scholarship ID133318. At the time this research was conducted, JR was funded by a Career Development and Future Leader Fellowship co-funded by the National Health and Medical Research Council and the National Heart Foundation. AR is funded by an NHMRC Principal Research Fellowship APP1124780. JR and AR are investigators on NHMRC program grant ID1052555.

\section{Conflicts of Interest}

SL is employed by the Better Health Company who are supported by the NSW Government to deliver the Go4Fun program. CR is the director, and CI-H is also employed by the NSW Office of Preventive Health, who manage the Go4Fun program.

\section{References}

1. World Health Organisation. Obesity and Overweight: Fact Sheet No. 311.

2. Singh AS, Mulder C, Twisk JWR, Van Mechelen W, Chinapaw MJM (2008) Tracking of childhood overweight into adulthood: a systematic review of the literature. Obes Rev 9: 474-488.

3. Daniels SR (2006) The consequences of childhood overweight and obesity. Future Child 16: 47-56.

4. Biro FM, Wien M (2010) Childhood obesity and adult morbidities. Am J Clin Nutr 91: 1499s-1505s.

5. Kamath CC, Vickers KS, Ehrlich A, McGovern JJ, Remberto Paulo VS, et al. (2008) Behavioral Interventions to Prevent Childhood Obesity: A Systematic Review and Metaanalyses of Randomized Trials. J Clin Endocr Metab 93: 4606-4615.

6. Epstein LH, Wrotniak BH (2010) Future directions for pediatric obesity treatment. Obesity 18: S8 - S12.

7. Commonwealth of Australia 2008.2007 Australian National Children's Nutrition and Physical Activity Survey - Main Findings.

8. National Health and Medical Research Council (2013) Clinical practice guidelines for the management of overweight and obesity in adults, adolescents and children in Australia. NHMRC : Melbourne, Australia.

9. National Institute for Health and Clinical Excellence (NICE) Guidance. (2006) Obesity: The Prevention, Identification, Assessment and Management of Overweight/Obesity in Adults \& Children.

10. Okely AD, Collins CE, Morgan PJ, Jones RA, Warren JM, et al. (2010) Multi-site randomized controlled trial of a child-centered physical activity program, a parent-centered dietary-modification program, or both in overweight children: The HIKCUPS study. J Pediatr 157: 388394.

11. Margarey AM, Perry RA, Baur LA, Steinbeck KS, Sawyer M, et al. (2001) A Parent-Led Family-Focused Treatment Program for Overweight Children Aged 5 to 9 Years: The PEACH RCT. Paediatrics 127: 214-222.

12. Spear BA, Barlow SE, Ervin C, Ludwig DS, Saelens BE, et al. (2007) Recommendations for treatment of child and adolescent overweight and obesity. Pediatrics 120: S254-S288.

13. Giles EL, Robalino S, McColl E, Sniehotta FF, Adams J (2014) The Effectiveness of Financial Incentives for Health Behaviour Change: Systematic Review and Meta-Analysis. PLOS One 9: e90347.

14. Mantzari E, Vogt F, Shemilt I, Wei Y, Higgins JP, et al. (2015) Personal financial incentives for changing habitual health-related behaviors: A systematic review and meta-analysis. Prev Med 75: 75-85. 
15. Mitchell MS, Goodman JM, Alter DA, John LK, Oh PI, et al. (2013) Financial incentives for exercise adherence in adults: systematic review and meta-analysis. Am J Prev Med 45: 658-667.

16. Paul Ebhohimhen V, Avenell A (2008) Systematic review of the use of financial incentives in treatments for obesity and overweight. Obes Rev 9: 355-367.

17. Purnell JQ Gernes R, Stein R, Sherraden MS, Knoblock Hahn A (2014) A systematic review of financial incentives for dietary behaviour change. J Acad Nutri Diet 114: 1023-1035.

18. Strohacker K, Galarraga O, Williams DM (2013) The Impact of Incentives on Exercise Behavior: A Systematic Review of Randomized Controlled Trials. Ann Behav Med: 1-8.

19. Sutherland K, Christianson JB, Leatherman S (2008) Impact of targeted financial incentives on personal health behaviour: a review of the literature. Med Care Res Rev 65: S65-78S.

20. Wall J, Mhurchu CN, Blakely T, Rodgers A, Wilton J (2006) Effectiveness of monetary incentives in modifying dietary behaviour: a review of randomised controlled trials. Nutr Rev 64: 518-531.

21. Enright G, Redfern J (2016) Summary of the evidence for the role of incentives in health-related behavior change: Implications for addressing childhood obesity. Annals of Public Health Research 3 1042.

22. Just DR, Price J (2013) Using Incentives to Encourage Healthy Eating in Children. J Hum Res 48: 855-872.

23. Loewenstein G, Price J, Volpp K (2015) Habit formation in children Evidence from incentives for healthy eating. J Health Econ 45: 47-54.

24. Hardman CA, Horne PJ, Fergus Lowe C (2011) Effects of rewards, peermodelling and pedometer targets on children's physical activity: a school-based intervention study. Psychol Health 26: 3-21.

25. Horne PJ, Hardman CA, Lowe CF, Rowlands AV (2009) Increasing children's physical activity: a peer modeling, rewards and pedometerbased intervention. Eur J Clin Nutr 63: 191

26. Horne PJ, Tapper K, Lowe CF, Hardman CA, Jackson MC, et al. (2004) Increasing children's fruit and vegetable consumption: A peer modeling and rewards-based intervention. Eur J Clin Nutr 58: 16491660 .

27. Morrill BA, Madden GJ, Wengreen HJ, Fargo JD, Aguilar SS (2015) A Randomised Controlled Trial of the Food Dudes Program: Tangible Rewards Are More Effective Than Social Rewards for Increasing Shortand Long-Term Fruit and Vegetable Consumption. J Acad Nutri Diet: S2212-2672.

28. Marchal B, Westhorp G, Wong G, Van Belle S, Greenhalgh T, et al. (2013) Realist RCTs of complex interventions - An oxymoron. Soc Sci Med 94: 124-128.

29. Moore GF, Audrey S, Barker M, Bond L, Bonell C, et al. (2015) Process evaluation of complex interventions: Medical Research Council guidance. BMJ 350: 1258

30. O'Cathain A, Goode J, Drabble SJ, Thomas KJ, Rudolph I, et al. (2014) Getting added value from using qualitative research with randomized controlled trials: a qualitative interview study. Trials 15: 215.

31. Patel B, Patel A, Jan S, Usherwood T, Harris M, et al (2014) Multifaceted quality intervention improvement intervention for CVD risk management in Australian primary health care: a protocol for a process evaluation. Implement Sci 9: 187.

32. Redfern J, Enright G, Raadsma S, Allman Farinelli M, Innes Hughes C, et al. (2016) Effectiveness of a behavioral incentive scheme linked to goal achievement: study protocol for a randomized controlled trial. Trials 17: 3 .
33. Sacher PM, Chadwick P, Wells JC, Williams JE, Cole TJ, et al. (2005) Assessing the acceptability and feasibility of the MEND Programme in a small group of obese 7-11-year-old children. J Hum Nutri Diet 18: 3-5.

34. Centres for Disease Control and Prevention, Division of Nutrition Physical Activity, and Obesity, Body Mass Index.

35. Virginia Braun, Victoria Clarke (2006) Using thematic analysis in psychology, Qualitative Research in Psychology 3(2): 77-101.

36. Enright G, Gyani A, Raadsma S, Allman-Farinelli M, Rissel C, et al. (2016) Evaluating factors influencing the delivery and outcomes of an incentive-based behaviour change strategy targeting child obesity: protocol for a qualitative process and impact evaluation. BMJ Open 6 : 1-10.

37. Michie S, Richardson M, Johnston M, Abraham C, Francis J, et al. (2013) The Behavior Change Technique Taxonomy (v1) of 93 Hierarchically Clustered Techniques: Building an International Consensus for the Reporting of Behavior Change Interventions. Ann Behav Med: 46: 81.

38. Tate DF, Lytle LA, Sherwood NE, Haire Joshu D, Matheson D, et al. (2016) Deconstructing interventions: approaches to studying behavior change techniques across obesity interventions. Transl Behav Med 6: 236.

39. Boutron I, Moher D, Altman DG, Schulz KF, CONSORT Group (2008) Extending the CONSORT statement to randomized trials of nonpharmacologic treatment: Explanation and elaboration. Ann Intern Med 148: 295-309.

40. Wilfley DE, Stein RI, Saelens BE, Mockus DS, Matt GE, et al. (2007) Efficacy of maintenance treatment approaches for childhood overweight: a randomized controlled trial. JAMA 298: 1661-1673.

41. Riley G (2016) The role of self-determination theory and cognitive evaluation theory in home education. Cogent Education 3: 1163651.

42. Anderson P, Harrison O, Cooper C, Jane Llopis E (2011) Incentives for Health. J Health Commun 16: 107-133.

43. Fryer R (2011) Financial Incentives and Student Achievement: Evidence from Randomized Trials. Quarterly Journal of Economics 126: $1755-1798$.

44. Gneezy U, Meier S, Rey Biel P (2011) When and why incentives (don't) work to modify behaviour. J Econ Perspect 25: 191-209.

45. Adriaanse MA, Vinkers CD, De Ridder DT, Hox JJ, De Wit, et al. (2011) Do implementation intentions help to eat a healthy diet? A systematic review and meta-analysis of the empirical evidence. Appetite 56: 183193.

46. Belanger Gravel A, Godin G, Amireault S (2013) A meta-analytic review of the effect of implementation intentions on physical activity. Health Psychol Rev 7: 23-54.

47. Gollwitzer PM, Sheeran P (2006) Implementation intentions and goal achievement: A meta-analysis of effects and processes. Adv exp soc psychol 38: 69-119.

48. Kivetz R, Urminsky O, Zheng Y (2006) The Goal-Gradient Hypothesis Resurrected: Purchase Acceleration, Illusionary Goal Progress, and Customer Retention. J Marketing Res 43: 39-58.

49. Harkin B, Webb TL, Chang BPI, Prestwich A, Conner M, et al. (2016) Does monitoring goal progress promote goal attainment? A metaanalysis of the experimental evidence. Psychol Bull 142: 198-229.

50. Hallsworth M, Berry D, Sanders M, Sallis A, King D, et al. (2015) Stating Appointment Costs in SMS Reminders Reduces Missed Hospital Appointments: Findings from Two Randomised Controlled Trials. PLOS ONE 10: e0141461. 
51. Ryan RM, Deci EL (2017) Self-determination theory: Basic psychological needs in motivation, development, and wellness. Guilford Publishing: New York, USA

52. Bandura A (1986) Social Foundations of Thought and Action: A Social Cognitive Theory. Prentice-Hall, Inc: New Jersey.

53. Deci EL, Ryan RM (1985) Cognitive evaluation theory. In Intrinsic Motivation and Self-Determination in Human Behavior. Springer: Boston, USA, pp: 87-112.

54. Deci EL, Ryan RM (1985) Toward an organismic integration theory. In Intrinsic Motivation and Self-Determination in Human Behavior. Springer: Boston, USA pp: 113-148.

55. Deci EL, Ryan RM (2000) The "what" and "why" of goal pursuits: Human needs and the self-determination of behavior. Psychol Inq 11: 227-268.
56. Oussedik E, Foy CG, Masicampo EJ, Kammrath LK, Anderson RE, et al. (2017) Accountability: a missing construct in models of adherence behavior and in clinical practice. Patient prefer adher 11: 1285-1294.

57. Redfern J, Enright G, Hyun K, Raadsma S, Farinelli MA, et al. (2019) Effectiveness of a behavioral incentive scheme linked to goal achievement in overweight children: a cluster randomized controlled trial. J Obesity and Diabetes 3: 1-8

58. Koestner R, Otis N, Powers T, Pelletier L, Gagnon H (2008) Autonomous Motivation, Controlled Motivation, and Goal Progress. J Pers: 12011230 .

59. Bailey ST (2008) Release Your Brilliance: The 4 Steps to Transforming Your Life and Revealing Your Genius to the World. Harper Collins: New York, USA.

\section{Your next submission with Juniper Publishers will reach you the below assets}

- Quality Editorial service

- Swift Peer Review

- Reprints availability

- E-prints Service

- Manuscript Podcast for convenient understanding

- Global attainment for your research

- Manuscript accessibility in different formats

( Pdf, E-pub, Full Text, Audio)

- Unceasing customer service

Track the below URL for one-step submission https://juniperpublishers.com/online-submission.php 